\title{
RISK-BASED REPLACEMENT STRATEGIES FOR DETERIORATING REINFORCED CONCRETE PIPES
}

Authors: Bryan Adey ${ }^{1}$, Olivier Bernard ${ }^{2}$ and Bruno Gerard ${ }^{2}$

${ }^{1}$ Division of Maintenance and Safety, Faculty of Architecture, and Civil and Environmental Engineering, Swiss Federal Institute of Technology, Switzerland, http://mcswww.epfl.ch

${ }^{2}$ Oxand S.A. - France, www.oxand.com

\begin{abstract}
Water distribution networks comprised of underground reinforced concrete pipes deteriorate with time due to environmental conditions, such as chloride-induced corrosion and differential soil movements [1]. If the pipes in these networks are not periodically replaced, they will eventually fail. Strategies for replacement for these pipes depend on 1) the risks associated with failure of a water distribution network, and 2) the costs associated with replacing the pipes, including the cost of removing existing pipes, the cost of the new pipes and the cost of service interruption if there is any temporary closure [2].
\end{abstract}

This paper gives an example of how predictive models of the deterioration of reinforced concrete pipes and the consequences of failure can be used to develop risk-based replacement strategies for underground reinforced concrete pipe networks. Replacement strategies are determined for a series network consisting of 1500 underground reinforced concrete pipes. The variation in cumulative overall costs of choosing the replacement times for each pipe group is shown, with and without discount rate.

This paper also shows the importance of correctly modelling the deterioration speed by analysing the example series network for three different deterioration speeds. The possible cost savings by accurately estimating the deterioration speed are shown. The main conclusion is that the use of accurate predictive models in the determination of replacement strategies for underground reinforced concrete pipe networks, the approach used by Oxand S.A, can result in significant savings in cumulative overall costs.

\section{Introduction}

Reinforced concrete pipes are often used in water distribution networks. These pipes deteriorate with time due to environmental conditions, such as chloride-induced corrosion and differential soil movements [1]. If they are not periodically replaced, they will eventually fail.

ADEY, Risk-based replacement strategies, $1 / 10$

Fax: 41216932873

E-mail: Bryan.adey@epfl.ch 
The risks associated with the failure of a water distribution network play a crucial role in the development of management strategies for these networks [3]. Risk is defined herein as the probability of failure multiplied by the consequences of failure, and the consequences of failure include the interruption of service until the network is restored to full operability and the damage that results from a burst such as the flooding of nearby buildings or roads.

One way to diminish risks related to water distribution networks is to replace deteriorated pipes [4]. There are, however, costs associated with replacing pipes, including the cost of removing the existing pipes, the cost of the new pipes and the cost of service interruption of any temporary closure, which mean that the owner of the network must carefully considered when to replace pipes. Optimal replacement strategies for the pipes must therefore be determined by minimising both the risks and the cost of replacement for the pipes in the network, as well as how these risks and costs change with time and the effectiveness of the replacement in reducing future risks. Optimal replacement strategies are herein referred to with the abbreviation for optimal management strategy, OMS. Replacement strategies are one type of management strategy.

\section{Predictive models of pipe deterioration}

Markov models and semi-Markov models can be used to model the deterioration of the pipes. Markov models are a commonly used, in management systems, to model the deterioration of infrastructure assets, such as pipes [5] and road bridges [6]. Semi-Markov models, however, have been used to incorporate changes in failure mode that may occur as a function of both time and the number of previous breaks [7]. As an unchanging failure mode is assumed in this example Markov models are considered adequate. In Markov models the condition ratings take the form of discrete states in order to reduce the complexity of associated with continuous ranking systems [6].

A Markov model describes a stochastic process where the conditional probability of any future event, such as being in condition state $j$, given any past event and the present state $X_{t}=i$, is independent of the past event and depends only on the present state. The conditional probabilities $P\left\{X_{t+1}=j \mid X_{t}=i\right\}$ are called transition probabilities. The transition probabilities in a Markov model can be determined using Poisson and negative binomial based regression techniques $[8,9]$ and can be correlated to actual deterioration models [10] albeit not perfectly. The possible transition probabilities are often shown in matrix form. The form of the Markov models used in this example (Eq. 1) is based on the five-state model shown in Fig 1.

\begin{tabular}{|c|c|c|c|c|c|}
\hline & $X_{1}$ & $X_{2}$ & $X_{3}$ & ${ }_{4} \quad X_{5}$ & \\
\hline$X_{1}$ & $1-\left(p_{12}+p_{13}+p_{14}+p_{15}\right)$ & $p_{12}$ & $p_{13}$ & $p_{14}$ & $p_{15}$ \\
\hline$X_{2}$ & & $1-\left(p_{23}+p_{24}+p_{25}\right)$ & $p_{23}$ & $p_{24}$ & $p_{25}$ \\
\hline$X_{3}$ & & & $1-\left(p_{34}+p_{35}\right)$ & $p_{34}$ & $p_{35}$ \\
\hline$X_{4}$ & & & & $1-p_{45}$ & $p_{45}$ \\
\hline$X_{5}$ & & & & & 1 \\
\hline
\end{tabular}


where $p_{i j}=$ transition probability from condition state $X_{i}$ in year t to condition state $X_{j}$ in year t +1 . For example, column 1 row 1 (in bold) shows the probability of being in condition state $X_{I}$ at $\mathrm{t}+1$ if the pipe is in condition state $X_{l}$ at time $\mathrm{t}$. Note the $p_{i j}=0$ for $\mathrm{i}>\mathrm{j}$. This imposes the constraint that pipes cannot improve in condition. Also $p_{55}=1$ because this is the worst possible condition state and it is an absorbing state, i.e. once a pipe has entered this state it cannot leave without an intervention.

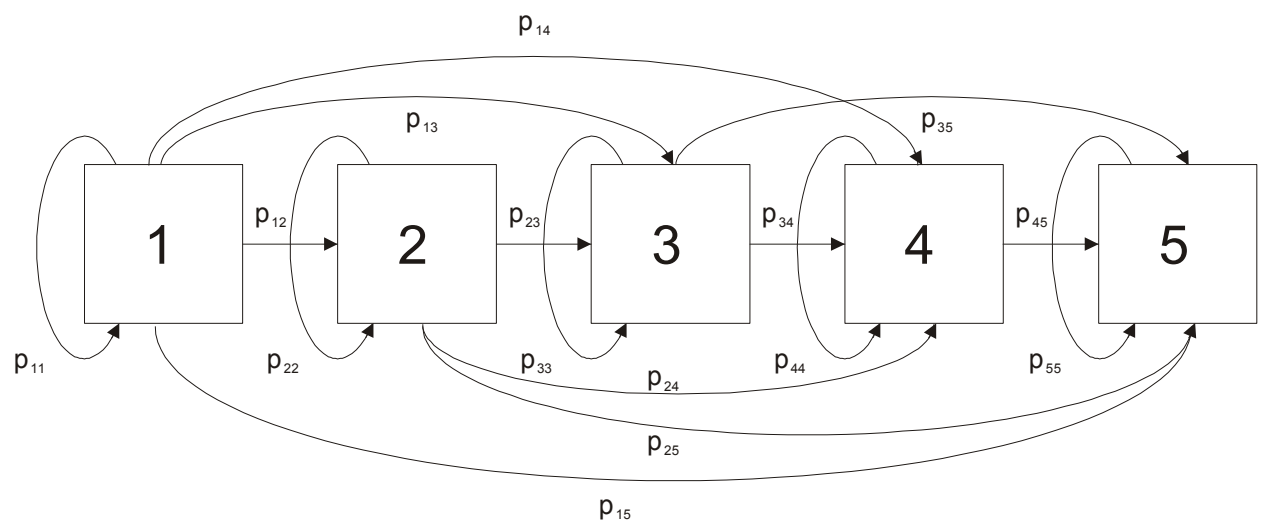

Figure 1: State transition diagram for five-state pipe example

The probability of being in condition state $X_{j}$, in year $\mathrm{t}+1$ can be determined through the application of the total probability theorem (Eq.2).

$p_{j}^{t+1}=\sum_{i=1}^{j} P_{i j} \cdot p_{i}^{t}$

where $p_{i}^{t}=$ the probability of being in condition state $X_{i}$, in year $\mathrm{t}$.

\section{Determination of risk-based optimal replacement strategies}

To determine risk-based OMS the optimisation problem is defined to minimise cumulative overall costs (both agency costs and risks) (Eq. 3).

Minimise $\lambda=c C_{a}+c C_{f}$

where:

$$
\begin{aligned}
& c C_{a}=\sum_{t=0}^{T}\left(C_{a}\right)_{t}=\text { cumulative agency costs; and } \\
& c C_{f}=\sum_{t=0}^{T}\left(P_{f s y s} \cdot C_{f}\right)_{t}=\text { cumulative risk } \\
& C_{a} \quad=\text { agency costs } \\
& C_{f} \quad=\text { failure costs } \\
& P_{f s y s}=\text { probability of failure of the system }
\end{aligned}
$$

ADEY, Risk-based replacement strategies, 3/10

Fax: 41216932873

E-mail: Bryan.adey@epfl.ch 


\section{Example network}

To illustrate how predictive models of the deterioration of reinforced concrete pipes and the consequences of failure can be used to develop risk-based replacement strategies an example pipe network consisting of 1500 pipes in series is used. The pipes in the network are classified into 5 different condition states (CS), which are defined in Table 1 [3]. It is assumed that inspection of the entire 1500 pipes is performed prior to the determination of the OMS, and that one quarter of the pipes (375) is in each of the condition states, i.e. there are 375 pipes initially in CS1, CS2, CS3 and CS4. All pipe deterioration is assumed to be the same and is described by Eq. 4.

Table 1: Condition states for underground reinforced concrete pipes[2]

\begin{tabular}{|c|l|}
\hline Condition state & Physical description \\
\hline 1 & Near perfect condition \\
\hline 2 & Some superficial deterioration \\
\hline 3 & Serious deterioration, requiring substantial maintenance \\
\hline 4 & $\begin{array}{l}\text { Level of deterioration affects the fabric of the asset, requiring major } \\
\text { reconstruction or refurbishment }\end{array}$ \\
\hline 5 & Level of deterioration is such to render the asset unserviceable \\
\hline
\end{tabular}

$$
\left\{\begin{array}{ccccc}
0.923 & 0.074 & 0.003 & 0 & 0 \\
0 & 0.9522 & 0.0478 & 0 & 0 \\
0 & 0 & 0.976 & 0.024 & 0 \\
0 & 0 & 0 & 0.998 & 0.002 \\
0 & 0 & 0 & 0 & 1
\end{array}\right\}
$$

The four possible interventions on the network are to replace all of the pipes that were initially in each condition state, i.e. CS4, CS3, CS2 and CS1 (Table 2). It is assumed that there can be only one intervention in each 5 -year period. The total time period investigated is 100 years. All interventions are assumed to cost $100 \mathrm{mu}$. It is assumed that if an intervention is not done and failure (defined as CS5) occurs, that the 6-meter long section of pipe where the leak occurs is replaced and the rest of the network is not. This has the effect of leaving the network in basically the same overall condition state, i.e. the probability of failure of this section of pipe is basically not changed, and the expected failure costs in the upcoming year therefore remain unchanged.

Table 2: Interventions

\begin{tabular}{|c|c|}
\hline Intervention & Pipes replaced \\
\hline 1 & Pipes initially in CS4 \\
\hline 2 & Pipes initially in CS3 \\
\hline 3 & Pipes initially in CS2 \\
\hline 4 & Pipes initially in CS1 \\
\hline
\end{tabular}

ADEY, Risk-based replacement strategies, 4/10

Fax: 41216932873

E-mail: Bryan.adey@epfl.ch 
The OMS's are determined for four interventions, i.e. only 4 interventions can be performed in the 100-year investigated time period. The cost of failure is $1000 \mathrm{mu}$, or 10 times the intervention cost. An algorithm using complete enumeration and dynamic programming was used to find the optimal solution.

\subsection{Optimal replacement strategies}

The OMS's are determined for the example network assuming discount rates of 0 and $10 \%$ (Table 3). The discount rate is the difference between the interest rate for borrowing money and the inflation rate. It is found that intervention 1 should be performed immediately, $\mathrm{t}_{0}$, and intervention 2 should be performed at $t_{3}$, regardless of the discount rate. This is because the risks associated with the pipes initially in CS4 and CS3 first become unacceptably high in years 0 and 3 , respectively, regardless of the discount rate.

Table 3: OMS's - example network

\begin{tabular}{|c|c|c|}
\hline & \multicolumn{2}{|l|}{ Optimal time interval } \\
\hline Intervention & Discount Rate $0 \%$ & Discount rate $10 \%$ \\
\hline 1 & $\mathrm{t}_{0}$ & $\mathrm{t}_{0}$ \\
\hline 2 & $\mathrm{t}_{3}$ & $\mathrm{t}_{3}$ \\
\hline 3 & $\mathrm{t}_{7}$ & $\mathrm{t}_{9}$ \\
\hline 4 & $\mathrm{t}_{9}$ & $\mathrm{t}_{14}$ \\
\hline
\end{tabular}

It is found that a discount rate of $10 \%$ pushes the optimal replacement times into the future. For example, the optimal time for intervention 3 if the discount rate is $0 \%$ is $\mathrm{t}_{7}$, and if the discount rate is $10 \%$ is $t_{9}, 10$ years later. This is because all of the future costs associated with failure are worth less.

\subsection{Variation of costs as a function of replacement time selected}

The possible variation of cumulative overall costs due to the variation of the four interventions in time, with a discount rate of 0 and $10 \%$, can be seen in Figures 2 and 3, respectively. In each of the four graphs, three of the intervention times are held constant and one is varied. For example, in Figure $2 b$ and $3 b$ the time of intervention 2 is varied from time interval 0 to time interval 19 while intervention 1,3 and 4 are performed at $t_{0}, t_{7}$ and $t_{9}$, respectively. The optimal time for the intervention varied is indicated with a large circle.

It can be seen that the choice of when to perform an intervention can result in significant differences in the cumulative overall costs when the discount rate is $0 \%$ (Figure 2). A delay of one time interval of intervention 1 , whose optimal time is in $t_{0}$, will result in an increase of $363 \mathrm{mu}$ (3.63 times the $C_{a}$ ) (Figure 2a). A delay of one time interval from the optimal time intervals of interventions 2, 3 and 4 will result in increased cumulative overall costs of $15 \mathrm{mu}$, $15 \mathrm{mu}$, and $3 \mathrm{mu}$, (Figures $2 \mathrm{~b}, 2 \mathrm{c}$, and $2 \mathrm{~d}$ ) respectively. If the interventions are performed one time interval earlier than the optimal time intervals, the increased cumulative overall costs will be $54 \mathrm{mu}, 10 \mathrm{mu}$, and $8 \mathrm{mu}$, respectively. The relatively small differences in cumulative overall costs indicate that there is a relatively large time period in which to perform one out four interventions without substantial penalty in overall costs. It should however be kept in mind that these are overall costs (agency costs + risks).

ADEY, Risk-based replacement strategies, 5/10

Fax: 41216932873

E-mail: Bryan.adey@epfl.ch 
The shapes of the cumulative overall cost curves shown in Figure 2 are due to the exponential characteristics of the probability of system failure equation (Eq. 5).

$$
P_{f s y s}=P_{f 1} \cdot P_{f 2} \cdot \ldots \ldots \cdot P_{f n}=P_{f}^{n}
$$

In Figure 2a there is essentially a linear relationship between cumulative overall costs and the time of intervention 1 . This is because the probability of system failure is essentially certain in each time interval and therefore for each time interval intervention 1 is displaced the cumulative overall costs are increased by the failure costs that will be incurred in the time interval where the intervention was to have been performed.

In Figures $2 b, c$ and $d$ there is an increasing relationship between cumulative overall costs and the time of interventions 2, 3 and 4, in each direction from the optimal intervention times. For example, moving intervention 3 (Figure 2c) from interval 14 to 15 results in a smaller increase in the cumulative overall costs than moving the intervention from interval 13 to interval 14 . This is because there not a certainty of system failure after intervention 1 is performed in $t_{0}$. Without a certainty of system failure the movement of the intervention time increases the probability of system failure exponentially (Eq.5).

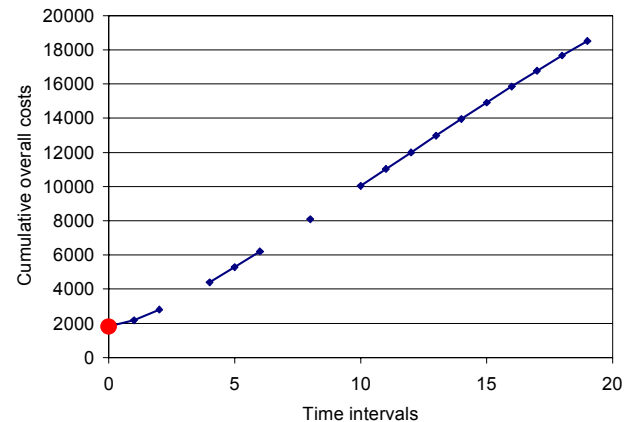

(a)

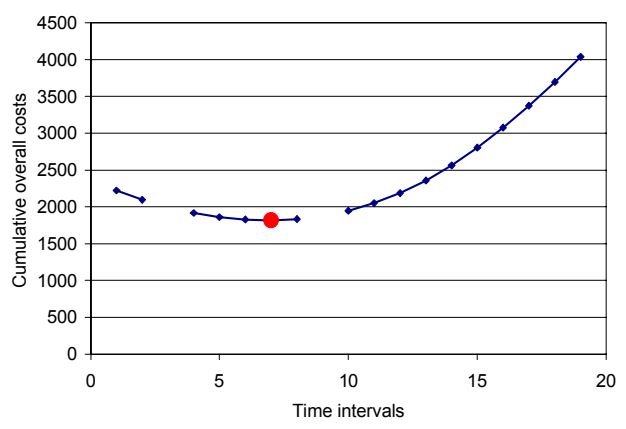

(c)

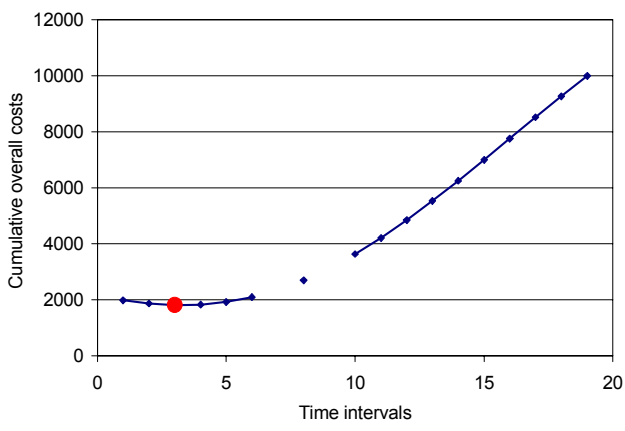

(b)

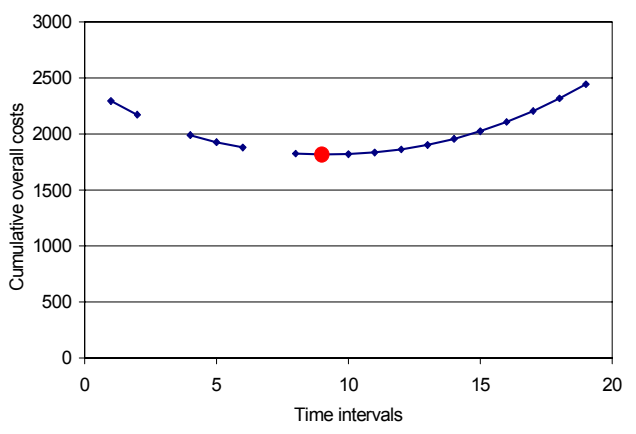

(d)

Figure 2: Variation in cumulative overall costs with discount rate $0 \%$ vs. the variation in time of (a) intervention 1, (b) intervention 2, (c) intervention 3, (d) intervention 4. 
The possible differences in the cumulative overall costs by varying the interventions over a 100 -year period, in present day dollars when the discount rate is $10 \%$, are $1099 \mathrm{mu}, 62 \mathrm{mu}$, $56 \mathrm{mu}$, and $55 \mathrm{mu}$ (Figure 3). The cumulative overall cost curves are asymptotic as the intervention is moved further into the future because future monetary units are worth increasingly less in the future, in present day monetary units. A delay of one time interval of intervention 1, whose optimal time is in $\mathrm{t}_{0}$, would result in an increase of $290 \mathrm{mu}\left(2.9 \times C_{a}\right)$. A delay of one time interval from the optimal time intervals of intervention 2, 3 and 4 will result in increased cumulative overall costs of $15 \mathrm{mu}, 15 \mathrm{mu}$, and $3 \mathrm{mu}$, (Figures $3 \mathrm{~b}, \mathrm{c}$ and d) respectively. If the interventions are performed one time interval earlier than the optimal time intervals for interventions 2,3 and 4 the increased cumulative overall costs will be $54 \mathrm{mu}$, $10 \mathrm{mu}$, and $8 \mathrm{mu}$, respectively. The exponential effects shown in Figure 2 are less apparent in Figure 3 due to the damping effect of the discount rate.

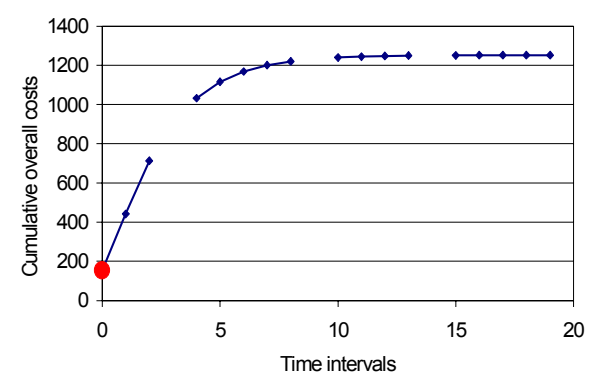

(a)

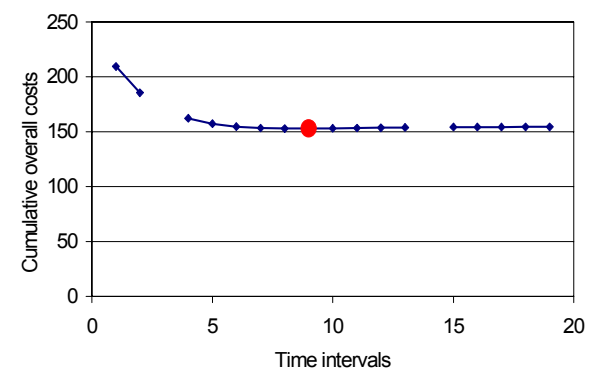

(c)

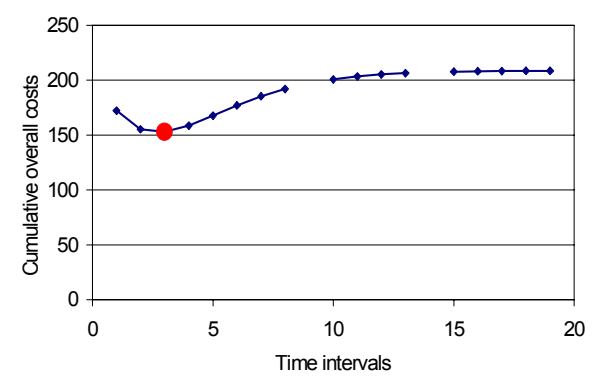

(b)

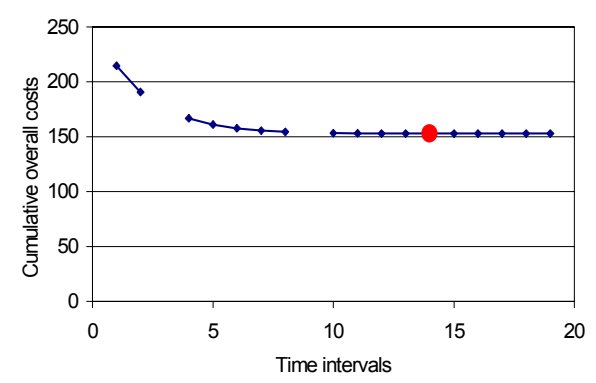

(d)

Figure 3. Variation in cumulative overall costs with discount rate $10 \%$ versus the variation in time of (a) intervention 1, (b) intervention 2, (c) intervention 3, (d) intervention 4. 
The OMS's shown in Table 3 illustrate that predictive models of deterioration can be used to determine risk-based replacement strategies for deteriorating reinforced concrete pipes. Comparison of these two OMS's as well as the possible variations in cumulative overall costs between them (Figures 2 and 3) shows that the consideration of a discount rate can push the optimal intervention times into the future. In section 5 the possible savings by accurately determining deterioration are shown.

\section{Accurate deterioration prediction}

To investigate the importance of accurate determination of deterioration speeds of underground reinforced concrete pipe networks, the OMS's, consisting of the first three interventions shown in Table 2, are determined for the example network using three different deterioration speeds, slow, medium and fast (Table 4). Slow, medium and fast deterioration speeds are defined in this example by Eqs. 5, 6 and 7, respectively. Slow deterioration is defined as having a $0.5 \%$ chance that a pipe will pass out of its condition state in one time interval. Medium deterioration is defined as having a $1 \%$ chance that a pipe will pass out of its condition state in one time interval. Fast deterioration is defined as having a $2 \%$ chance that a pipe will pass out of its condition state in one time interval.

Table 4: OMS's - series pipe network - slow/medium/fast deterioration

\begin{tabular}{|c|c|c|c|}
\hline & \multicolumn{3}{|c|}{ Year of intervention } \\
\hline Intervention & Slow & Medium & Fast \\
\hline 1 & 0 & 0 & 0 \\
\hline 2 & 5 & 2 & 1 \\
\hline 3 & 19 & 12 & 7 \\
\hline
\end{tabular}

Slow deterioration: $\quad\left\{\begin{array}{ccccc}0.995 & 0.005 & 0 & 0 & 0 \\ 0 & 0.995 & 0.005 & 0 & 0 \\ 0 & 0 & 0.995 & 0.005 & 0 \\ 0 & 0 & 0 & 0.995 & 0.005 \\ 0 & 0 & 0 & 0 & 1\end{array}\right\}$
Medium deterioration: $\left\{\begin{array}{ccccc}0.990 & 0.010 & 0 & 0 & 0 \\ 0 & 0.990 & 0.010 & 0 & 0 \\ 0 & 0 & 0.990 & 0.010 & 0 \\ 0 & 0 & 0 & 0.990 & 0.010 \\ 0 & 0 & 0 & 0 & 1\end{array}\right\}$
Fast deterioration: $\left\{\begin{array}{ccccc}0.980 & 0.020 & 0 & 0 & 0 \\ 0 & 0.980 & 0.020 & 0 & 0 \\ 0 & 0 & 0.980 & 0.020 & 0 \\ 0 & 0 & 0 & 0.980 & 0.020 \\ 0 & 0 & 0 & 0 & 1\end{array}\right\}$


To show the possible savings in cumulative overall costs by accurately estimating the deterioration speed, the cumulative overall costs are calculated for the example network with each deterioration speed and each replacement strategy. A discount rate of $8 \%$ is used. Figure $4 \mathrm{a}, \mathrm{b}$ and $\mathrm{c}$ show the cumulative overall costs for the example network if the deterioration speed is slow, medium and fast, and the OMS's determined assuming slow, medium and fast deterioration are implemented, respectively. The time interval in which the interventions are to be performed are indicated with a large circle.

In this example if the actual deterioration is slow, knowing that it is slow rather than average or fast, can result in up to a $30 \%$ difference in cumulative overall costs over a 100 -year period. If the actual deterioration is average, knowing that it is average rather than slow or fast, can result in a difference of up to $18 \%$ in cumulative overall costs over a 100 -year period. If the actual deterioration is fast, knowing it is fast rather than average or slow, can result in up to an $86 \%$ difference in cumulative overall costs over a 100 -year period. These differences could be larger if the strategies weren't optimal and the deterioration speed was not estimated correctly.

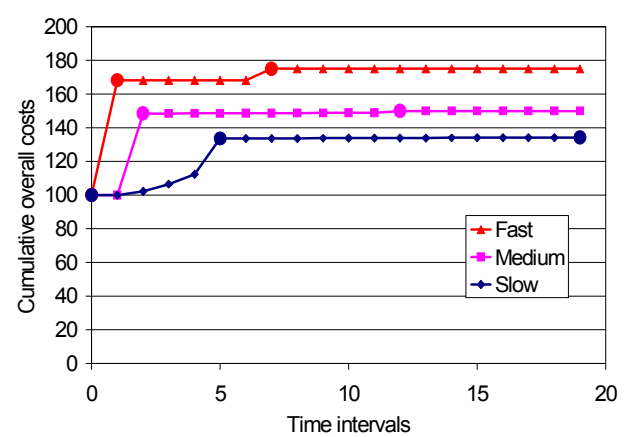

(a)

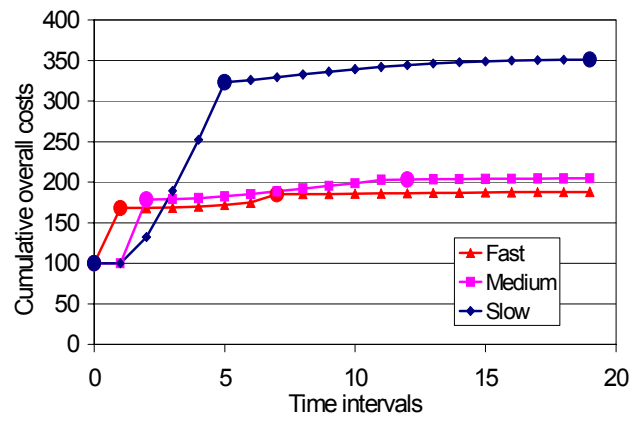

(c)

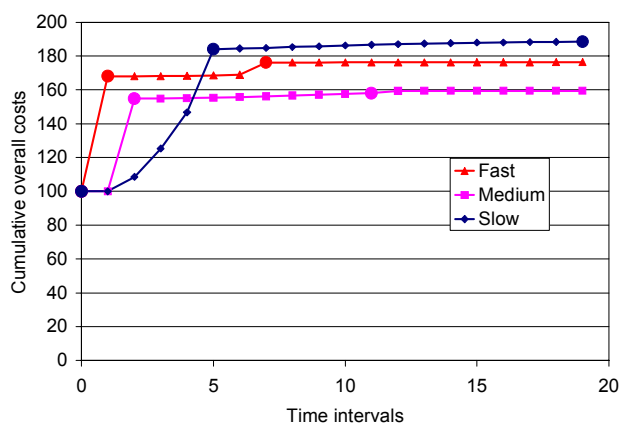

(b)

Figure 4. Cumulative overall costs vs. time (a) slow deterioration, (b) medium deterioration, (c) fast deterioration 


\section{Conclusions}

This example has shown that:

1) Predictive deterioration models can be used in the determination of risk-based replacement strategies for underground reinforced concrete pipe networks; and

2) Accurate deterioration prediction is necessary in order to find optimal replacement strategies for underground reinforced concrete pipe networks. This requires a rigorous initial investigation when determining the optimal replacement strategy and may also warrant a continual monitoring of the network throughout its service life.

Future work needs to concentrate on the accuracy of the deterioration models used in predicting future deteriorating of underground reinforced concrete pipes.

\section{References}

1 Peter-Lazar, I., Heinfling G., Gerard B., and Marchand J., 'Application of probabilistic methods to the analysis of the behaviour of reinforced concrete structures affected by steel corrosion', in 'Concrete durability', Proceedings of a CANMET Conference, Barcelona, June, 2000, 557-572.

2 Luong, H.T., and Fujiwara, O., 'Fund allocation for pipe repair maintenance in water distribution networks', European Journal of Operational Research 136 (2002), 403-421.

3 Adey, B., Hajdin R., and Brühwiler E., 'A system approach to the reduction of damage costs due to natural hazards', in 'Towards a Better Built Environment - Innovation, Sustainability, Information Technology', Proceedings of an IABSE Symposium, Melbourne, Australia, Sept., 2002, CD.

4 Creig-Smith, S., 'To refurbish or replace water pipelines, that is the question', Engineering failure analysis, 8 (2001) 107-112.

5 Micevski T., Kuczera G., and Coombes P., 'Markov Model for Storm Water Pipe Deterioration', Journal of Infrastructure Systems 8 (2) (2002) 49-56.

6 Cambridge Systematics, 'Pontis Release 3.2 - Users Manual', American Association of State Highway and Transportation Officials Inc., Washington D.C. (1997).

7 Li, D., and Haimes, Y.Y., 'Optimal Maintenance Related Decision Making for Deteriorating Water Distribution Systems 1. Semi-Markovian Model for a Water Main', Water Resources Research 28 (4) (1992) 1053-1061.

8 Madanat, S., Mishalani, R., and Ibrahim, W.H.W., 'Estimation of infrastructure transition probabilities', Journal of Infrastructure Engineering 1 (2) (1995) 120-125.

9 Madanat, S., and Ibrahim, W.H.W., 'Poisson regression models of infrastructure transition probabilities', Journal of Transportation Engineering 121 (3) (1995) 267-272.

10 Roelfstra, G., 'A model of condition evolution of concrete highway bridges' (in French), Ph.D. thesis 2310, The Swiss Federal Institute of Technology, Lausanne, Switzerland, 2001.

ADEY, Risk-based replacement strategies, 10/10

Fax: 41216932873

E-mail: Bryan.adey@epfl.ch 
